GRASAS Y ACEITES, 63 (4),

OCTUBRE-DICIEMBRE, 403-410, 2012,

ISSN: 0017-3495

DOI: $10.3989 /$ gya. 058212

\title{
Influencia del proceso de maduración del fruto en la calidad sensorial de aceites de oliva virgen de las variedades Picual, Hojiblanca y Picudo
}

\author{
Por B. Jiménez Herrera ${ }^{1, *}$, A. Rivas Velasco², A. Sánchez-Ortiz ${ }^{3}$, M.L. Lorenzo Tovar², \\ M. Úbeda Muñoz ${ }^{1}$, R.M. Callejón ${ }^{4}$ y E. Ortega Bernaldo de Quirós ${ }^{5}$ \\ ${ }^{1}$ IFAPA Centro «Cabra», Antigua Ctra. Cabra-Doña Mencía, Km. 2,5, 14940 Cabra, Córdoba \\ ${ }^{2}$ Dpto. de Nutrición y Bromatología, Universidad de Granada \\ ${ }^{3}$ IFAPA Centro «Venta del Llano», Ctra. Bailén-Motril, Km. 18.5, 23620, Mengibar, Jaén \\ ${ }^{4}$ Dpto. de Nutrición y Bromatología, Toxicología y Medicina Legal, Universidad de Sevilla \\ ${ }^{5}$ Dpto. de Edafología y Química Agrícola, Universidad de Granada \\ Autor para la correspondencia: brigida.jimenez@juntadeandalucia.es
}

\section{RESUMEN}

Influencia del proceso de maduración del fruto en la calidad sensorial de aceites de oliva virgen de las variedades Picual, Hojiblanca y Picudo.

La calidad sensorial del aceite de oliva virgen (AOV) está estrechamente relacionada con la variedad y el grado de maduración de la aceituna. El objetivo del presente trabajo fue investigar la influencia del grado de maduración sobre el perfil sensorial de aceites de oliva virgen monovarietales con el fin de establecer el momento óptimo de recolección. Los frutos de tres variedades diferentes, Picual, Picudo y Hojiblanca fueron recolectados en nueve etapas de maduración diferentes. Los parámetros de calidad fueron evaluados y las características organolépticas se determinaron por un panel de cata. Los resultados muestran que los parámetros analíticos disminuyeron ligeramente en todas las variedades. Para cada variedad se describe la evolución de las características organolépticas de los aceites de oliva virgen así como sus flavores típicos. En todas las variedades estudiadas, los atributos «frutado» (afrutado) y «amargo» disminuyeron durante la maduración, por el contrario el atributo «dulce» se incrementó. Los resultados mostrados pueden ser de gran utilidad para proveer información sobre la evolución de la calidad sensorial de los aceites de oliva virgen durante la maduración para obtener aceites basados en las preferencias del mercado.

PALABRAS CLAVE: Aceite de oliva virgen - Calidad sensorial - Flavor - Maduración del fruto - Preferencias del mercado - Variedades de aceitunas.

\section{SUMMARY}

Influence of fruit maturation process on the sensory quality of virgin olive oils from Picual, Hojiblanca and Picudo cultivars.

The sensory quality of virgin olive oil is closely correlated with the cultivar and the degree of ripening of the olive fruit. The aim of the present work was to investigate the influence of ripening degree on sensory profile of monovarietal virgin olive oils (VOO) in order to establish an optimum harvesting time. Fruit obtained from three different cultivars, Picual, Picudo and Hojiblanca were picked at nine different stages of ripeness. The quality parameters were evaluated and the sensory characteristics were determinate by a sensor panel. The analytical parameters decrease slightly in all cultivars.
The evolution of the organoleptic characteristics of the virgin olive oil is reported and typical flavors are described for each cultivar. In all studied cultivar, "fruity" and "bitter" attributes decreased during the ripening and conversely "sweet" attribute increased. The results showed in this work could be considered useful for providing information about the evolution of sensory quality of virgin olive oils during ripening to obtain those based on market preferences.

KEY-WORDS: - Flavor - Ripening - Sensory quality Virgin olive oil - Market preferences - Olive varieties.

\section{INTRODUCCIÓN}

La valoración sensorial es el principal parámetro de calidad apreciado por el consumidor de aceite de oliva virgen (AOV), siendo completado con los parámetros físico-químicos que se recogen en el Reglamento CEE no 2568/91 con posteriores modificaciones y anexos. Múltiples factores influyen en la calidad organoléptica y en las propiedades del AOV obtenido, entre los que se encuentran los factores agronómicos: variedad, suelo, riego, control de plagas y enfermedades que junto con la recolección van a ser determinantes para la obtención de frutos sanos de una madurez óptima que nos proporcionaran aceites de máxima calidad. Además, los factores postcosecha y tecnológicos, como transporte, almacenamiento, lavado, molienda, batido, etc., inciden muy directamente en las características organolépticas y en la composición química de los aceites obtenidos (Jiménez y Carpio, 2008). El estado de maduración de la aceituna es uno de los factores más importantes asociados a la calidad sensorial del aceite de oliva virgen (Youssef et al., 2010). Aparte de los conocidos efectos de la maduración de la aceituna sobre rendimiento graso, deterioro del fruto, color, etc., el factor maduración debe ser un criterio prioritario para definir la calidad organoléptica del aceite ya que, en función de ese estado, la composición química (triglicéridos y compuestos minoritarios) del aceite elaborado sufre alteraciones que, según la 
época de recolección del fruto, superan a las producidas por el factor variedad (Sánchez Casas et al., 2006; Inglese, 1994; Pinatel, 1996).

El desarrollo y posterior maduración del fruto es un proceso largo que dura entre 35 y 40 semanas. La lignificación del endocarpo se produce entre las 10 y las 12 semanas después de floración (SDF), y es en ese momento cuando se inicia la biosíntesis y acumulación de aceite, tanto en el mesocarpo como en la semilla (Rapoport, 2001). El proceso de maduración comienza aproximadamente a las 28 SDF y se caracteriza por un cambio del color del fruto que vira de verde a morado, hasta alcanzar un color prácticamente negro al final de la maduración (Sánchez, 1994). El proceso de desarrollo y maduración del fruto es resultado de una combinación de sucesos bioquímicos y fisiológicos que tienen lugar bajo un estricto control genético pero con influencia de factores medioambientales, edad del olivo o estado del árbol (Connor y Fereres, 2005).

Varios autores han estudiado estos cambios metabólicos que ocurren durante la maduración de la aceituna y que afectan a las características sensoriales del aceite obtenido. En este sentido numerosas evidencias experimentales apoyan la existencia de una relación inversa entre maduración del fruto y contenido de polifenoles y compuestos volátiles (Salvador et al., 2001; Bonoli et al., 2004; Rotondi et al., 2004; Gómez-Rico et al., 2006; Youseff et al., 2010; Martínez Nieto et al., 2010). Ambos determinan en buena medida, el característico sabor y aroma del AOV, respectivamente. Sin embargo, hay escasez de estudios que relacionen estos compuestos químicos con los descriptores sensoriales, que son en última estancia los que determinan la calidad del AOV que el consumidor percibe.

Por tanto, de todo lo anteriormente descrito se desprende la importancia de la determinación del momento óptimo de recolección de la aceituna, que tradicionalmente ha sido establecida en algunas regiones productoras de AOV en base al calendario. Por ello, el objetivo del presente trabajo de investigación es profundizar en el conocimiento del efecto del índice de madurez del fruto sobre la calidad sensorial de aceites de diferentes variedades producidas en Andalucía. Para ello se han seleccionado las variedades Picual, Hojiblanca y Picudo, que presentan diferentes patrones de maduración con objeto de determinar el momento óptimo de la recolección de la aceituna para que el aceite obtenido sea de máxima calidad sensorial. Los resultados obtenidos permitirán identificar que descriptores sensoriales están más relacionados con el momento óptimo de recolección de la aceituna y con los parámetros de calidad reglamentada para el AOV.

\section{MATERIALES Y MÉTODOS}

\subsection{Caracterización de la zona}

El presente estudio se llevó a cabo en la finca experimental «La Mina» ubicada en el centro que el Instituto de Investigación y Formación Agraria y Pesquera (IFAPA) posee en la localidad de Cabra, ciudad situada al sur de la provincia de Córdoba, a una distancia de $72 \mathrm{~km}$ de ésta, y en las estribaciones de las sierras Subbéticas. La zona presenta un clima mediterráneo continental lo que proporciona unos inviernos suaves y veranos secos. Las precipitaciones se distribuyen de otoño a primavera, estimándose la media anual entre unos $700 \mathrm{~mm}$ en las zonas bajas y unos $1.000 \mathrm{~mm}$ en las sierras. La nieve aunque no frecuente, no es del todo extraña en invierno. La temperatura media anual es de aproximadamente $17^{\circ} \mathrm{C}$, pudiéndose llegar $\mathrm{a}-4^{\circ} \mathrm{C}$ en los meses de invierno y a $43^{\circ} \mathrm{C}$ en los meses de verano.

Los suelos se caracterizan por ser pocos profundos y estar desarrollados principalmente sobre rocas calizas y silíceas. Presentan una textura franca, el pH es básico, con valores comprendidos entre 8 y 8.5. La conductividad eléctrica es baja así como también los es el contenido en materia orgánica y la relación carbono/nitrógeno.

\subsection{Material vegetal}

El ensayo se realizó en las variedades Picual, Hojiblanca y Picudo de la finca. El marco de plantación es de $7 \mathrm{~m}$ entre filas y $7 \mathrm{~m}$ entre árboles. La edad de los árboles es de 27 años los de las variedades «Picual» y «Hojiblanca» y de 16 años los de la variedad «Picuda». Se seleccionaron 10 árboles de cada variedad. La toma de muestras se llevó a cabo semanalmente, iniciándose el 25/10/2010 y teniendo que finalizar el 20/12/2010 debido a la falta de aceituna en el árbol por la caída natural de ésta y para evitar el riesgo de heladas que afectan a la calidad sensorial. La nomenclatura utilizada para las fechas de recolección de las muestras en las variedades analizadas queda recogida en la Tabla 1.

\subsection{Diseño experimental, evaluación de la madurez del fruto y extracción del AOV}

Cada replicado de muestra fue de $7 \mathrm{Kg}$, de los que se tomaron al azar 100 frutos para calcular el

Tabla 1

Fecha de recogida en el año 2010 de las aceitunas analizadas

\begin{tabular}{cc}
\hline Numeración & Fecha de recogida \\
\hline I & $25 / 10$ \\
II & $02 / 11$ \\
III & $08 / 11$ \\
IV & $15 / 11$ \\
V & $22 / 11$ \\
VI & $29 / 11$ \\
VII & $06 / 12$ \\
VIII & $13 / 12$ \\
IX & $20 / 12$ \\
\hline
\end{tabular}


índice de madurez (I.M.), utilizado habitualmente en las almazaras (Ferreira, 1979). Al resto de muestra se le extrajo el aceite con el sistema Abencor (MC2 Ingeniería y Sistemas SL, Sevilla, España) (Martínez et al., 1975), determinándose en éste el valor de los parámetros legalmente establecidos para evaluar su grado de calidad comercial, incluyendo su análisis sensorial. El experimento se llevó a cabo por triplicado.

\subsection{Parámetros físico-químicos}

En cada una de las muestras de aceite se determinaron los parámetros físico-químicos elementales como grado de acidez (G.A), índice de peróxidos (I.P), y absorbancia a la radiación ultravioleta $\mathrm{K}_{270}$ y $\mathrm{K}_{232}$, siguiendo el método oficial del Anexo Il del Reglamento de la CE 2568/91. El índice de amargor $\mathrm{K}_{225}$, se determinó por separación en fase sólida (SPE) de los compuestos fenólicos y medida a $225 \mathrm{~nm}$ del extracto obtenido (Gutiérrez et al., 1992).

\subsection{Análisis organoléptico}

Esta evaluación se realizó con un panel de ocho catadores selecionados y entrenados (Galán- Soldevilla, H y Ruíz Pérez- Cacho), pertenecientes al Panel de Cata de la Denominación de Origen (DOP) Priego de Córdoba. La valoración organoléptica se llevó a cabo según el Reglamento (CE) № 640/2008 de la Comisión de 4 de julio de 2008 y el método de «Valoración Organoléptica que opta a una Denominación de Origen » (Norma COI/t.20/ Doc. no 22/ Noviembre de 2005), ya que estas variedades están amparadas en la DOP Priego de Córdoba. La cata de los aceites se realizó según la norma COI/t. 20/ Doc. no 13/Rev. 1 Noviembre de 1996, utilizando la hoja de perfil del Reglamento (CE) № 640/2008. A continuación, se valoró las sensaciones olfativas directas o retronasales de los atributos «frutado, verde hoja/hierba, manzana, almendra, plátano, higuera, alcachofa, otros positivos"; las sensaciones gustativas de los descriptores «amargo y dulce» y las sensaciones táctiles o quinesteticas mediante el atributo «picante», empleando para ello la ficha de cata en donde los catadores tenían que marcar las intensidades de los diferentes descriptores en una escala continua del 1 al 10 (Norma COI/t.20/ Doc. no 5/ Noviembre de 2005). Además, los catadores evaluaron globalmente los aceites con una puntuación entre 1 y 10.

\subsection{Análisis estadístico}

Para establecer diferencias entre medias se aplicó el test de Tukey, utilizando el programa SPSS 15.0 (SPSS Inc., Chicago, IL). Las diferencias se consideraron estadísticamente significativas cuando la probabilidad era superior al $95 \%$ $(p<0.05)$. Se calculó el coeficiente de correlación de Pearson entre las puntuaciones globales de cada aceite y las puntuaciones obtenidas en los distintos atributos sensoriales.

\section{RESULTADOS Y DISCUSIÓN}

Cuando se inició el trabajo, todas las variedades estudiadas presentaron unos índices de madurez bajos comprendidos entre 0 y 1 (Tabla 2 ), que aumentaron significativamente de forma progresiva cada semana, aunque siguiendo patrones diferentes en cada variedad. La variedad más temprana es decir la que maduró antes, fue la Picual, mientras que las más tardías en la maduración fueron la Hojiblanca y Picuda, ambas muy igualadas.

La variación en los índices de calidad reglamentada: acidez, índice de peróxidos y absorción en el UV se muestran en la Tabla 2. Todas las muestras estudiadas estuvieron dentro de los límites establecidos por la UE para los aceites de oliva vírgenes extra (Reglamento CE/1989/2003).

De los resultados analíticos estudiados cabe destacar los obtenidos para el $\mathrm{K}_{225}$ por su estrecha relación con el descriptor sensorial «amargo». De este modo los datos muestran que el aceite de las variedades Picual y Picuda presentaron los niveles más elevados, en concordancia con los resultados del análisis sensorial (Tablas 3-5). Durante el proceso de maduración del fruto se produjo un descenso significativo en el amargor de los aceites, tendencia que se repite en todas las variedades estudiadas.

Los resultados obtenidos en el análisis sensorial se muestran en las Tablas 3-5. En primer lugar, destacaron los descriptores «frutado», "amargo", "picante» y "dulce» que fueron percibidos en todas las muestras de las tres variedades estudiadas. Los datos reflejan que los aceites que se obtienen de aceitunas recolectadas en estado de maduración más bajo, presentaron valores más acusados y estadísticamente significativos en los atributos «amargo y picante», respecto a los obtenidos en los aceites con estados de madurez más altos. Estos atributos presentaron una mayor variabilidad de un estado a otro de madurez, coincidiendo con los trabajos realizados por Sánchez-Casas et al., (2006) para variedades de aceitunas producidas en Extremadura.

Los atributos sensoriales «amargo y picante» son debidos a la activación de los receptores del sabor y de las terminaciones del nervio trigémino asociadas a los receptores del sabor de las papilas gustativas, sensitivas a los estímulos químicos. En los aceites de oliva vírgenes estas sensaciones están relacionadas con la presencia de compuestos fenólicos y pueden persistir por un tiempo elevado después de la deglución, mostrando un claro efecto en intensidad y duración que puede variar entre los aceites de oliva y puede afectar la aceptación por el consumidor (Caporale et al., 2006).

En nuestro caso, la intensidad del atributo "picante" se percibió de forma diferente en los aceites de las distintas variedades. La variedad Picual fue 
Tabla 2

Variación de los parámetros físico-químicos de los aceites durante el proceso de maduración del fruto

\begin{tabular}{|c|c|c|c|c|c|c|c|c|c|}
\hline & I & II & III & IV & $\mathbf{V}$ & VI & VII & VIII & IX \\
\hline \multicolumn{10}{|l|}{ PICUAL } \\
\hline I.M. & $0.4^{i}$ & $1.2^{\mathrm{h}}$ & $2.2^{\mathrm{g}}$ & $2.3^{f}$ & $2.8^{\mathrm{e}}$ & $3.1^{d}$ & $3.5^{\mathrm{c}}$ & $4.2^{b}$ & $4.7^{\mathrm{a}}$ \\
\hline G.A (\% oleico) & $0.2^{\mathrm{a}}$ & $0.2^{\mathrm{a}}$ & $0.1^{\mathrm{b}}$ & $0.1^{\mathrm{b}}$ & $0.1^{\mathrm{b}}$ & $0.2^{\mathrm{a}}$ & $0.2^{\mathrm{a}}$ & $0.2^{\mathrm{a}}$ & $0.2^{\mathrm{a}}$ \\
\hline I.P $\left(\right.$ meq $\left.\mathrm{O}_{2} / \mathrm{Kg}\right)$ & $5.8^{\mathrm{a}}$ & $5.6^{\mathrm{b}}$ & $5.1^{\mathrm{d}}$ & $5.2^{c}$ & $4.7^{\mathrm{e}}$ & $4.1^{\dagger}$ & $3.9^{9}$ & $3.2^{h}$ & $1.9^{\mathrm{i}}$ \\
\hline $\mathrm{K}_{232}$ & $1.7^{\mathrm{b}}$ & $1.7^{\mathrm{b}}$ & $1.6^{\mathrm{c}}$ & $1.8^{\mathrm{a}}$ & $1.6^{c}$ & $1.5^{\mathrm{d}}$ & $1.5^{\mathrm{d}}$ & $1.4^{\mathrm{e}}$ & $1.3^{f}$ \\
\hline $\mathrm{K}_{270}$ & $0.2^{\mathrm{a}}$ & $0.2^{\mathrm{a}}$ & $0.2^{\mathrm{a}}$ & $0.2^{\mathrm{a}}$ & $0.1^{\mathrm{b}}$ & $0.2^{\mathrm{a}}$ & $0.1^{\mathrm{b}}$ & $0.1^{\mathrm{b}}$ & $0.1^{\mathrm{b}}$ \\
\hline $\mathrm{K}_{225}$ & $0.4^{\mathrm{a}}$ & $0.3^{b}$ & $0.3^{b}$ & $0.4^{\mathrm{a}}$ & $0.4^{\mathrm{a}}$ & $0.4^{\mathrm{a}}$ & $0.3^{b}$ & $0.3^{b}$ & $0.3^{b}$ \\
\hline \multicolumn{10}{|l|}{ HOJIBLANCA } \\
\hline I.M. & $0.4^{i}$ & $0.5^{\mathrm{h}}$ & $1.4^{\mathrm{g}}$ & 1. $8^{f}$ & $2.5^{\mathrm{e}}$ & $3.6^{d}$ & 3. $9^{c}$ & $4.8^{\mathrm{a}}$ & $4.4^{b}$ \\
\hline G.A (\% oleico) & $0.2^{\mathrm{a}}$ & $0.1^{\mathrm{b}}$ & $0.2^{\mathrm{a}}$ & $0.1^{\mathrm{b}}$ & $0.1^{\mathrm{b}}$ & $0.2^{\mathrm{a}}$ & $0.2^{\mathrm{a}}$ & $0.1^{\mathrm{b}}$ & $0.2^{\mathrm{a}}$ \\
\hline I.P (meq O $2 / \mathrm{Kg})$ & $7.6^{\mathrm{b}}$ & $7.7^{\mathrm{a}}$ & $6.5^{\mathrm{d}}$ & $7.4^{\mathrm{c}}$ & $5.7^{e}$ & $3.7^{\mathrm{g}}$ & $5.7^{e}$ & $6.6^{d}$ & $4.2^{f}$ \\
\hline $\mathrm{K}_{232}$ & $1.5^{\mathrm{b}}$ & $1.6^{\mathrm{a}}$ & $1.5^{\mathrm{b}}$ & $1.5^{\mathrm{b}}$ & $1.4^{\mathrm{c}}$ & $1.5^{\mathrm{b}}$ & $1.5^{\mathrm{b}}$ & $1.3^{\mathrm{d}}$ & $1.4^{\circ}$ \\
\hline $\mathrm{K}_{270}$ & $0.2^{\mathrm{a}}$ & $0.2^{\mathrm{a}}$ & $0.1^{\mathrm{b}}$ & $0.1^{\mathrm{b}}$ & $0.1^{\mathrm{b}}$ & $0.2^{\mathrm{a}}$ & $0.1^{\mathrm{b}}$ & $0.1^{\mathrm{b}}$ & $0.1^{\mathrm{b}}$ \\
\hline $\mathrm{K}_{225}$ & $0.2^{b}$ & $0.2^{b}$ & $0.3^{\mathrm{a}}$ & $0.3^{\mathrm{a}}$ & $0.3^{\mathrm{a}}$ & $0.2^{b}$ & $0.2^{b}$ & $0.3^{\mathrm{a}}$ & $0.1^{\mathrm{c}}$ \\
\hline \multicolumn{10}{|l|}{ PICUDA } \\
\hline I.M & $0.9^{i}$ & $1.2^{\mathrm{h}}$ & $1.7^{\mathrm{g}}$ & $2.1^{\mathrm{e}}$ & $1.9^{f}$ & $2.2^{d}$ & $2.8^{\mathrm{c}}$ & $4.8^{\mathrm{a}}$ & $3.8^{\mathrm{b}}$ \\
\hline G.A (\% oleico) & $0.2^{\mathrm{a}}$ & $0.1^{\mathrm{b}}$ & $0.2^{\mathrm{a}}$ & $0.1^{\mathrm{b}}$ & $0.2^{\mathrm{a}}$ & $0.2^{\mathrm{a}}$ & $0.1^{\mathrm{b}}$ & $0.2^{\mathrm{a}}$ & $0.2^{\mathrm{a}}$ \\
\hline I.P (meq O $2 / \mathrm{Kg})$ & $6.8^{\mathrm{a}}$ & $4.5^{\mathrm{e}}$ & $5.0^{\mathrm{c}}$ & $6.2^{\mathrm{b}}$ & $4.7^{\mathrm{d}}$ & $4.1^{\dagger}$ & $3.9^{9}$ & $3.2^{\mathrm{h}}$ & $2.9^{i}$ \\
\hline $\mathrm{K}_{232}$ & $2.0^{\mathrm{a}}$ & $1.9^{b}$ & $2.0^{\mathrm{a}}$ & $2.1^{\mathrm{a}}$ & $2.1^{\mathrm{a}}$ & $2.1^{\mathrm{a}}$ & $2.2^{\mathrm{a}}$ & $2.2^{\mathrm{a}}$ & $1.6^{\mathrm{c}}$ \\
\hline $\mathrm{K}_{270}$ & $0.2^{\mathrm{a}}$ & $0.2^{\mathrm{a}}$ & $0.2^{\mathrm{a}}$ & $0.1^{\mathrm{b}}$ & $0.1^{\mathrm{b}}$ & $0.2^{\mathrm{a}}$ & $0.2^{\mathrm{a}}$ & $0.1^{\mathrm{b}}$ & $0.1^{\mathrm{b}}$ \\
\hline $\mathrm{K}_{225}$ & $0.5^{\mathrm{a}}$ & $0.5^{\mathrm{a}}$ & $0.4^{\mathrm{b}}$ & $0.5^{\mathrm{a}}$ & $0.3^{c}$ & $0.2^{d}$ & $0.3^{\mathrm{c}}$ & $0.3^{c}$ & $0.3^{\mathrm{c}}$ \\
\hline
\end{tabular}

Los datos son expresados como valores medios de 3 experimentos independientes. Distintas letras en la misma línea representa que existen diferencias significativas entre las medias. Discriminación de medias con el test de Tukey con un nivel de significación del 95\% ( $p<0.05)$. I.M. Índice de Madurez; G.A Grado de Acidez; I.P Índice de Peróxidos.

la que presentó los valores más bajos en este atributo, oscilando entre 3,2 y 2,2. Esta variedad, además alcanzó una mayor intensidad en el atributo "amargo", por lo que fue este atributo el que persistió en el tiempo tras su ingesta. Los aceites obtenidos en la variedad Picuda se caracterizaron por un picor que se percibió más en la garganta, iniciándose con una alta intensidad de 5.3, disminuyendo la intensidad ligeramente durante el proceso de maduración de los frutos hasta 3.1. En la variedad Hojiblanca la sensación quinestética del «picante»se percibió en boca (no en garganta), siendo esta sensación muy leve al inicio, la cual fue incrementándose y persistiendo en el tiempo. Sin embargo, la intensidad de dicho descriptor en esta variedad disminuyó a lo largo de los muestreos, iniciándose las campañas con una intensidad de 4.7 y finalizando con una intensidad de 2.5 .

Con respecto al atributo «frutado», destaca el grado de intensidad de este atributo, que alcanzó los valores más altos de intensidad en los muestreos II y III de la variedad Picual con una puntuación máxima de 7.2. Estos resultados están en concordancia con los trabajos de Rotondi et al.,
(2004) en variedades de aceitunas italianas, que muestran como el atributo «frutado» alcanza sus valores máximos en niveles de maduración más bajos. Asimismo, durante el proceso de maduración del fruto se produjo un aumento del descriptor «dulce» de los aceites, tendencia que se repite en todas las variedades estudiadas. Otros autores como Hermoso et al., (1991) y Beltran et al., (2008), también evidenciaron que los descriptores de los aceites se ven fuertemente influenciados por el estado de maduración del fruto, obteniéndose aceites menos amargos ó más dulces en frutos más maduros, disminuyendo los valores medios del atributo amargo en el proceso de maduración.

Respecto a los demás atributos sensoriales, los que fueron percibidos en el análisis sensorial de los aceites procedentes de la variedad Picual fueron «verde hoja, verde hierba, almendra, manzana e higuera», destacando sobre todo el «verde hoja», seguido del "verde hierba, manzana e higuera». Los aceites obtenidos de aceitunas de la variedad Hojiblanca se caracterizaron por los descriptores «verde hoja, verde hierba, manzana, almendra y alcachofa», siendo el más acentuado "verde hierba» y siguiéndo- 
Tabla 3

Variación de los atributos sensoriales de los aceites procedentes de la variedad Picual durante el proceso de maduración del fruto

\begin{tabular}{|c|c|c|c|c|c|c|c|c|c|}
\hline & I & II & III & IV & V & VI & VII & VIII & IX \\
\hline Frutado & $6.2^{b}$ & $7.2^{\mathrm{a}}$ & $6.2^{b}$ & $6.1^{\mathrm{b}}$ & $6.2^{b}$ & $6.2^{b}$ & $6.2^{b}$ & $5.6^{c}$ & $5.7^{c}$ \\
\hline Amargo & $4.5^{\mathrm{a}}$ & $3.9^{c}$ & $4.2^{b}$ & $3.6^{c}$ & $2.4^{d}$ & $4.2^{\mathrm{b}}$ & $4.2^{b}$ & $4.2^{\mathrm{b}}$ & $3.9^{c}$ \\
\hline Picante & $3.1^{\mathrm{a}}$ & $3.0^{\mathrm{a}}$ & $3.2^{\mathrm{a}}$ & $2.5^{\mathrm{b}}$ & $3.2^{\mathrm{a}}$ & $2.7^{\mathrm{b}}$ & $2.6^{\mathrm{b}}$ & $2.4^{\mathrm{bc}}$ & $2.2^{\mathrm{c}}$ \\
\hline Dulce & $2.0^{\mathrm{bc}}$ & $1.7^{\mathrm{c}}$ & $2.3^{\mathrm{b}}$ & $2.3^{b}$ & $2.3^{b}$ & $1.7^{\mathrm{c}}$ & $1.8^{\mathrm{C}}$ & $2.3^{b}$ & $2.6^{a}$ \\
\hline Verde hoja & $3.8^{b}$ & $3.6^{b}$ & $3.5^{\mathrm{bc}}$ & $4.1^{\mathrm{a}}$ & $3.1^{\mathrm{C}}$ & $3.3^{c}$ & $3.2^{\mathrm{c}}$ & $3.2^{c}$ & $3.2^{c}$ \\
\hline Verde hierba & $2.6^{c}$ & $2.7^{\mathrm{bc}}$ & $1.5^{\mathrm{d}}$ & $3.1^{\mathrm{a}}$ & $2.6^{c}$ & $2.3^{\mathrm{c}}$ & $1.9^{\mathrm{d}}$ & $1.9^{d}$ & $2.6^{c}$ \\
\hline Manzana & $2.6^{\mathrm{b}}$ & $1.6^{\mathrm{de}}$ & $3.1^{\mathrm{a}}$ & $1.5^{\mathrm{d}}$ & $1.4^{\mathrm{e}}$ & 2. $8^{\mathrm{b}}$ & $2.4^{\mathrm{C}}$ & $1.7^{\mathrm{d}}$ & $2.0^{\mathrm{d}}$ \\
\hline Almendra & n.d & $3.1^{\mathrm{a}}$ & n.d & $3.1^{\mathrm{a}}$ & $2.7^{\mathrm{b}}$ & $0.8^{d}$ & $1.6^{c}$ & n.d & $1.3^{\mathrm{c}}$ \\
\hline Plátano & n.d & 3.53 & n.d & n.d & n.d & n.d & n.d & n.d & n.d \\
\hline Higuera & $2.1^{\mathrm{b}}$ & n.d & $2.0^{\mathrm{c}}$ & $2.1^{\mathrm{b}}$ & $2.1^{\mathrm{b}}$ & $2.3^{\mathrm{a}}$ & $2.2^{\mathrm{a}}$ & $2.2^{\mathrm{a}}$ & $2.0^{\mathrm{C}}$ \\
\hline Alcachofa & n.d & n.d & n.d & n.d & n.d & n.d & n.d & n.d & n.d \\
\hline Otros positivos & $1.1^{d}$ & $4.2^{\mathrm{a}}$ & $1.6^{\mathrm{c}}$ & $4.1^{\mathrm{a}}$ & $4.1^{\mathrm{a}}$ & $2.3^{b}$ & $2.4^{\mathrm{b}}$ & n.d & $1.2^{\mathrm{d}}$ \\
\hline Puntuación & $7.6^{\mathrm{b}}$ & $8.1^{\mathrm{a}}$ & $7.6^{b}$ & $7.5^{\mathrm{b}}$ & $7.5^{\mathrm{b}}$ & $7.6^{\mathrm{b}}$ & $7.6^{\mathrm{b}}$ & $7.3^{\mathrm{c}}$ & $7.4^{\mathrm{c}}$ \\
\hline
\end{tabular}

n.d: no detectado. Los datos son expresados como valores medios de 3 experimentos independientes. Distintas letras en la misma línea representan diferencias significativas entre las medias para $\mathrm{p} \leq 0.05$.

le «manzana». Por último, en los aceites que se extrajeron de la variedad Picuda, los descriptores identificados fueron el «verde hierba, verde hoja, manzana y almendra», encontrándose en mayor intensidad el "verde hierba y almendra». En general, en las variedades estudiadas se apreció un descenso en los valores de los atributos indicados a medida que avanza la época de recolección, a excepción del atributo «manzana» en la variedad Picuda que aumentó en estados de maduración más avanzados, y el «almen- dra» que alcanzó sus valores máximos a un nivel intermedio de índice de madurez.

Se calculó el coeficiente de correlación de Pearson entre las puntuaciones globales de cada aceite y las puntuaciones obtenidas en los distintos atributos sensoriales para cada variedad estudiada. Se obtuvo una correlación significativa entre los atributos sensoriales «frutado» y «verde hierba» en la variedad Picuda $(r=0.937)$ y «frutado» con «verde hierba» $(r=0.7083)$ y «alcachofa» $(r=0.929)$

Tabla 4

Variación de los atributos sensoriales de los aceites procedentes de la variedad Hojiblanca durante el proceso de maduración del fruto

\begin{tabular}{|c|c|c|c|c|c|c|c|c|c|}
\hline & I & II & III & IV & v & VI & VII & VIII & IX \\
\hline Frutado & $6.4^{\mathrm{b}}$ & $6.2^{\mathrm{b}}$ & $6.7^{\mathrm{a}}$ & $6.2^{\mathrm{b}}$ & $6.3^{b}$ & $6.6^{\mathrm{a}}$ & $6.7^{\mathrm{a}}$ & $6.2^{b}$ & $5.7^{\circ}$ \\
\hline Amargo & $3.0^{\mathrm{a}}$ & $2.6^{\mathrm{b}}$ & $3.2^{\mathrm{a}}$ & $2.6^{\mathrm{b}}$ & $2.3^{\mathrm{d}}$ & $2.4^{\mathrm{c}}$ & $2.4^{\mathrm{c}}$ & $1.8^{\mathrm{e}}$ & $1.9^{\mathrm{e}}$ \\
\hline Picante & $4.7^{\mathrm{c}}$ & $5.6^{b}$ & $6.1^{\mathrm{a}}$ & $4.6^{c}$ & $3.2^{\mathrm{e}}$ & $3.7^{d}$ & $3.1^{\mathrm{e}}$ & $3.1^{\mathrm{e}}$ & $2.5^{\dagger}$ \\
\hline Dulce & $2.4^{\mathrm{b}}$ & $2.9^{a}$ & $2.2^{\mathrm{b}}$ & $1.9^{c}$ & $2.9^{a}$ & $2.3^{\mathrm{b}}$ & $2.4^{\mathrm{b}}$ & $2.8^{\mathrm{a}}$ & $2.7^{\mathrm{a}}$ \\
\hline Verde hoja & $3.0^{\mathrm{a}}$ & $2.2^{\mathrm{b}}$ & $2.3^{\mathrm{b}}$ & $1.7^{\mathrm{c}}$ & $2.2^{\mathrm{b}}$ & $1.7^{\mathrm{c}}$ & $1.7^{\mathrm{c}}$ & $1.8^{\mathrm{c}}$ & $1.4^{\mathrm{d}}$ \\
\hline Verde hierba & $4.2^{b}$ & $4.1^{\mathrm{b}}$ & $5.1^{\mathrm{a}}$ & $2.9^{d}$ & $4.2^{\mathrm{b}}$ & $4.3^{\mathrm{b}}$ & $4.2^{b}$ & $4.2^{\mathrm{b}}$ & $3.4^{\mathrm{c}}$ \\
\hline Manzana & $3.2^{\mathrm{a}}$ & $2.0^{\mathrm{c}}$ & $3.1^{\mathrm{a}}$ & $3.1^{\mathrm{a}}$ & $3.1^{\mathrm{a}}$ & - & $0.6^{d}$ & $2.2^{b c}$ & $2.3^{\mathrm{bc}}$ \\
\hline Almendra & $2.2^{\mathrm{a}}$ & $1.8^{\mathrm{b}}$ & $2.1^{\mathrm{a}}$ & $2.1^{\mathrm{a}}$ & $2.2^{\mathrm{a}}$ & $1.7^{\mathrm{b}}$ & $2.3^{\mathrm{a}}$ & $2.3^{\mathrm{a}}$ & $1.3^{\mathrm{c}}$ \\
\hline Plátano & n.d & n.d & n.d & n.d & n.d & n.d & n.d & n.d & n.d \\
\hline Higuera & n.d & n.d & n.d & n.d & n.d & n.d & n.d & n.d & n.d \\
\hline Alcachofa & n.d & n.d & n.d & $2.1^{\mathrm{a}}$ & n.d & $2.3^{\mathrm{a}}$ & $2.3^{\mathrm{a}}$ & $2.2^{\mathrm{a}}$ & $1.3^{b}$ \\
\hline Otros positivos & $4.1^{\mathrm{a}}$ & $3.1^{\mathrm{b}}$ & $3.1^{\mathrm{b}}$ & n.d & n.d & $2.2^{c}$ & $2.3^{\mathrm{c}}$ & $2.3^{c}$ & $2.4^{\mathrm{C}}$ \\
\hline Puntuación & $7.7^{\mathrm{ab}}$ & $7.6^{\mathrm{b}}$ & $7.9^{\mathrm{a}}$ & $7.6^{\mathrm{b}}$ & $7.7^{\mathrm{ab}}$ & $7.8^{\mathrm{a}}$ & $7.8^{\mathrm{a}}$ & $7.6^{\mathrm{b}}$ & $7.3^{\mathrm{c}}$ \\
\hline
\end{tabular}

n.d: no detectado. Los datos son expresados como valores medios de 3 experimentos independientes. Distintas letras en la misma línea representan diferencias significativas entre las medias para $\mathrm{p} \leq 0.05$. 
Tabla 5

Variación de los atributos sensoriales de los aceites procedentes de la variedad Picuda durante el proceso de maduración del fruto

\begin{tabular}{|c|c|c|c|c|c|c|c|c|c|}
\hline & I & II & III & IV & V & VI & VII & VIII & IX \\
\hline Frutado & $5.6^{c}$ & $5.1^{d}$ & $6.7^{\mathrm{a}}$ & $6.3^{\mathrm{ab}}$ & $6.1^{\mathrm{b}}$ & $5.6^{c}$ & $5.5^{c}$ & $5.4^{\mathrm{cd}}$ & $5.3^{d}$ \\
\hline Amargo & $3.3^{b}$ & $4.6^{a}$ & $3.6^{b}$ & $2.8^{\mathrm{c}}$ & $3.6^{b}$ & $2.3^{d}$ & $2.5^{\mathrm{cd}}$ & $2.4^{\mathrm{d}}$ & $2.2^{d}$ \\
\hline Picante & $5.3^{\mathrm{a}}$ & $3.8^{c}$ & $5.1^{\mathrm{a}}$ & $4.5^{\mathrm{b}}$ & $3.2^{d}$ & $3.1^{\mathrm{e}}$ & $3.5^{\mathrm{cd}}$ & $3.3^{d}$ & $3.1^{\mathrm{e}}$ \\
\hline Dulce & $1.7^{\mathrm{b}}$ & $1.7^{\mathrm{b}}$ & $1.1^{\mathrm{d}}$ & $1.5^{\mathrm{c}}$ & $2.2^{\mathrm{a}}$ & $1.7^{\mathrm{b}}$ & $1.7^{\mathrm{b}}$ & $2.4^{\mathrm{a}}$ & $2.2^{\mathrm{a}}$ \\
\hline Verde hoja & $3.2^{b}$ & $3.5^{\mathrm{a}}$ & $2.3^{d}$ & $2.6^{c}$ & $2.7^{\mathrm{c}}$ & $1.7^{\mathrm{e}}$ & $1.2^{f}$ & n.d & n.d \\
\hline Verde hierba & $3.7^{c}$ & $1.5^{f}$ & $5.1^{\mathrm{a}}$ & $5.1^{\mathrm{a}}$ & $4.2^{b}$ & $3.2^{d}$ & $2.9^{e}$ & $2.6^{\mathrm{e}}$ & $2.8^{\mathrm{e}}$ \\
\hline Manzana & n.d & $1.4^{\mathrm{bc}}$ & $1.4^{\mathrm{c}}$ & $2.1^{\mathrm{a}}$ & $0.7^{d}$ & $2.1^{\mathrm{a}}$ & $1.7^{b}$ & $1.9^{\mathrm{ab}}$ & $1.7^{\mathrm{b}}$ \\
\hline Almendra & $3.2^{b}$ & $0.7^{e}$ & $2.6^{c}$ & $4.1^{\mathrm{a}}$ & $2.4^{\mathrm{c}}$ & $1.1^{d}$ & $2.3^{\mathrm{c}}$ & $3.2^{b}$ & $3.2^{b}$ \\
\hline Plátano & n.d & n.d & n.d & n.d & n.d & n.d & n.d & n.d & n.d \\
\hline Higuera & n.d & n.d & n.d & n.d & n.d & n.d & n.d & n.d & n.d \\
\hline Alcachofa & n.d & n.d & n.d & n.d & n.d & n.d & n.d & n.d & n.d \\
\hline Otros positivos & $3.1^{\mathrm{b}}$ & $1.1^{\mathrm{d}}$ & $4.2^{\mathrm{a}}$ & $4.1^{\mathrm{a}}$ & $4.2^{\mathrm{a}}$ & $2.1^{\mathrm{b}}$ & $1.2^{\mathrm{c}, \mathrm{d}}$ & $1.5^{\mathrm{c}}$ & n.d \\
\hline Puntuación & $7.3^{c}$ & $7.1^{\mathrm{d}}$ & $7.9^{\mathrm{a}}$ & $7.7^{\mathrm{b}}$ & $7.6^{\mathrm{b}}$ & $7.3^{c}$ & $7.2^{c}$ & $7.2^{c}$ & $7.2^{\mathrm{c}}$ \\
\hline
\end{tabular}

n.d: no detectado. Los datos son expresados como valores medios de 3 experimentos independientes. Distintas letras en la misma línea representan diferencias significativas entre las medias para $p \leq 0.05$.

en la variedad Hojiblanca. Asimismo, se encontró una correlación significativa entre el atributo «amargo» con «picante» $(r=0850)$ en la variedad Hojiblanca y «amargo» con «verde hoja» $(r=0.794)$ en la variedad Picuda. Por el contrario, no se observaron correlaciones significativas en la variedad Picual.

De forma global la evolución de la valoración sensorial nos indica que la puntuación total está influenciada por el índice de madurez, obteniéndose valores más elevados en el segundo muestreo en la variedad Picual y en el tercer muestreo en la Hojiblanca y Picuda. Asimismo, según los resultados obtenidos y tras hacer una correlación entre la puntuación total de la calidad sensorial y los descriptores encontrados, el atributo «frutado» está directamente correlacionado con la calidad organoléptica en la variedad Picual $(r=0.978)$, Hojiblanca $(r=0.983)$ y Picuda $(r=0.995)$. En la Tabla 6 se describen las características organolépticas más destacables de las variedades estudiadas.

\section{CONCLUSIONES}

Se ha comprobado que el perfil sensorial característico del aceite de cada variedad estudiada es modulado a lo largo de la maduración del fruto, permitiendo establecer una fecha óptima de reco-

Tabla 6

Características organolépticas de las variedades estudiadas

\begin{tabular}{|c|c|c|}
\hline Picual & Hojiblanca & Picuda \\
\hline $\begin{array}{l}\text { Frutado de aceituna verde hoja, } \\
\text { hierba, higuera, con notas frutales } \\
\text { de manzana, su entrada en boca es } \\
\text { dulce y almendrado. A medida que } \\
\text { pasa a la parte posterior, se percibe } \\
\text { un amargor medio y un picante más } \\
\text { ligero con un retrogusto a hoja de } \\
\text { olivo. } \\
\text { Estos aceites entan un amplio } \\
\text { periodo óptimo de recolección, con } \\
\text { puntuaciones organolépticas } \\
\text { elevadas y leves modificaciones en } \\
\text { el perfil sensorial, destacando las } \\
\text { notas a higuera en los estadios } \\
\text { finales de la maduración. }\end{array}$ & $\begin{array}{l}\text { Frutado de aceituna verde hierba, con } \\
\text { toques herbáceos de hoja de olivo, } \\
\text { notas frutales anzana y almendra, su } \\
\text { entrada en boca es dulce, almendrado } \\
\text { a medida que pasa a la parte posterior, } \\
\text { se percibe un amargor ligero y un } \\
\text { picante suave que se hace más intenso } \\
\text { y persistente con el paso del tiempo, } \\
\text { con un retrogusto alcachofa. } \\
\text { Al igual que la variedad Picual presenta } \\
\text { un mplio periodo óptimo de recolección, } \\
\text { con puntuaciones organolépticas } \\
\text { elevadas y leves modificaciones en el } \\
\text { perfil sensorial, destacando de manera } \\
\text { significativa la disminución del picante } \\
\text { a lo largo de la maduración. }\end{array}$ & $\begin{array}{l}\text { Frutado de aceituna verde hierba, con } \\
\text { toques herbáceos de hoja de olivo, } \\
\text { notas frutales anzana y almendra, su } \\
\text { entrada en boca es dulce, } \\
\text { almendrado a medida que pasa a la } \\
\text { parte posterior, se percibe un amargor } \\
\text { ligero, con intensidad media de } \\
\text { picante que se percibe en la garganta, } \\
\text { el ogusto es almendrado. } \\
\text { Presenta un periodo óptimo de } \\
\text { recolección situado entre las fases III } \\
\text { y V de muestreo, correspondiendo } \\
\text { con un índice de madurez cercano } \\
\text { al } 2 \text {, a partir del cual los atributos } \\
\text { organolépticos pierden intensidad. }\end{array}$ \\
\hline
\end{tabular}


lección de la aceituna que permite obtener aceites de mayor calidad sensorial. Las variedades Picual y Hojiblanca presentan un amplio período óptimo de recolección del fruto, con puntuaciones organolépticas elevadas y leves modificaciones en el perfil sensorial, destacando la disminución del picante en Hojiblanca. En la variedad Picuda el momento óptimo de recolección correspondería entre el III y V muestreo equivalente a un índice de madurez cercano al 2.

De cada variedad se pueden obtener aceites con diferentes intensidades en sus atributos ya que a medida que avanza el periodo de maduración los aceites obtenidos son más pobres y apagados tanto en aroma como en sabor. Los resultados sensoriales obtenidos en el presente trabajo pueden ser considerados de gran utilidad para proveer información sobre la evolución de la calidad sensorial a lo largo de la maduración del fruto. De este modo, se pueden obtener una variada gama de aceites de categoría virgen extra acorde a los gustos y exigencias de los consumidores.

\section{AGRADECIMIENTOS}

Los autores quieren expresar su agradecimiento a los catadores del panel de cata perteneciente a la Denominación de Origen (DOP) Priego de Córdoba por su colaboración en la evaluación sensorial de las muestras.

\section{REFERENCIAS}

Beltrán G, Uceda M, Hermoso M, Frías L. 2008. El Cultivo del Olivo. Junta de Andalucía-Consejería de Agricultura y Pesca and Ed. Mundi-Prensa. Madrid, Barcelona, México.

Beltrán G, Jiménez A, Aguilera MP, Uceda M. 2000. Análisis mediante HPLC de la fracción fenólica del aceite de oliva virgen de la variedad Arbequina. Relación con la medida del amargor K225 y la estabilidad. Grasas Aceites 51, 320-324.

Bonoli M, Bendini A, Cerretani L, Lercker G, Gallina T. 2004. Qualitative and semiquantitative analysis of phenolic compounds in extra virgin olive oils as a function of the ripening degree of olive fruits by different analytical techniques. J. Agric. Food Chem. 52, 7026-7032.

Caporale G, Policastro S, Carducci A, Monteleone E. 2006. Consumer expectations for sensory propierties in virgin olive oils. Food Qual. Prefer. 17, 116-125.

Connor, D. J. y Fereres, E. (2005). The physiology of adaptation and yield expression in olive. Horticultural Reviews 31, 155-229.

Consejo Oleícola Internacional 1996(COI/t.20/Doc. № 13/ Rev. 1). Metodología general para la valoración organoléptica del Aceite de Oliva Virgen.

Consejo Oleícola Internacional 2005 (COI/t.20/Doc. no 22). Método de valoración organoléptica del Aceite de Oliva Virgen Extra que opta a una Denominación de Origen.

Ferreira J. 1979. Explotaciones olivareras colaboradoras, $\mathrm{n}$ 5 . Ministerio de Agricultura, Madrid.
Galán-Soldevilla H, Ruiz Pérez-Cacho P. 2012. Panel training programme for the protected designation of origin «Aceituna Aloreña de Malaga». Grasas Aceites 63, 109-117.

Gómez-Rico A, Salvador MD, La Greca M, Fregapane G. 2006. Phenolic and volatile compounds of extra virgin olive oil (Olea europaea L.Cv. Cornicabra) with regard to fruit ripening and irrigation management. $J$. Agric. Food Chem. 54, 7130-7136.

Gómez-Rico A, Fregapane G, Salvador MD. 2008. Effect of cultivar and ripening on minor components in Spanish olive fruits and theri corresponding virgin olive oils. Food Res. Int. 41, 433-440.

Gracia S, Royo A, Guillen M. 2004. Composición química de aceites de las variedades Arbequina y Empeltre cultivadas en regadío. Grasas Aceites 51, 320-324.

Gutiérrez F, Perdiguero S, Gutiérrez R, Olías JM. 1992. Evaluation of the bitter taste in virgin olive oil. J. Am. Oil Chem. Soc. 69, 394-395.

Gutiérrez F, Jiménez B, Ruiz A, Albi MA. 1999. Effect of olive ripeness on the oxidative stability of virgin olive oil extracted from the varieties Picual and Hojiblanca and on the different component involved. J. Agric. Food Chem. 47, 121-127.

Hermoso M, Uceda M, García-Ortiz A, Morales J, Frías L, Fernández A. 1991. Elaboración de aceite de oliva de calidad. Colección: Apuntes, no 5/91. Dirección General de Investigación, Tecnología y Formación Agroalimentaria y Pesquera. Consejería de Agricultura y Pesca de la Junta de Andalucía, Sevilla, 173.

Inglese P. 1994. La influencia de la variedad en las características cualitativas del aceite de oliva. Olivae 54,42-47.

Jiménez B, Carpio A. 2008. La cata de aceites: Aceite de oliva virgen, en: Características organolépticas y análisis sensorial. Junta de Andalucía, Instituto de Investigación y Formación Agraria y Pesquera, Sevilla, 145.

Martinez Nieto L, Hodaifa G, Lozano JL. 2010. Changes in phenolic compounds and Rancimat stability of olive oils from varieties of olives at different stages of ripeness. J. Sci. Food Agric. 90, 2393-2398.

Martínez JM, Muñoz E, Alba J, Lanzon A. 1975. Informe sobre la utilización del analizador de rendimientos «Abencor». Grasas Aceites 26, 379-385.

Pinatel C. 1996. Variabilite organoleptique des huiles d'olive en fonction de la maturité et des techniques culturales. OCL 6, 80-83.

Rapoport HF. 2001. Botánica y Morfología, en: El Cultivo del Olivo. Mundiprensa, 35-60.

Reglamento (CE) 2568/ 1991 de la Comisión relativo a las características de los aceites de oliva y de los aceites de orujo de oliva y sobre sus métodos de análisis.

Reglamento (CE) 1989/ 2003 de la Comisión que modifica el Reglamento (CE) 2568/ 1991 relativo a las características de los aceites de oliva y de los aceites de orujo de oliva y sobre sus métodos de análisis.

Reglamento (CE) 640/ 2008 de la Comisión que modifica el Reglamento (CE) 2568/ 1991 relativo a las características de los aceites de oliva y de los aceites de orujo de oliva y sobre sus métodos de análisis.

Rotondi A, Bendini A, Cerretani L, Mari M, Kercker G, Gallina Toschi T. 2004. Effect of olive Ripening Degree on the Oxidative Stabity and Organoleptic Prop- 
erties of Cv. Nostrava di Brisighella Extra Virgin Olive Oil. J. Agric. Food Chem. 52, 3649-3654.

Sánchez Casas J, De Miguel C, Osorio E, Marín J, Gallardo L, Martínez M. 2006. Calidad sensorial de aceites de oliva virgen procedentes de variedades de aceitunas producidas en Extremadura. Grasas Aceites 57, 313-318.

Sánchez J. 1994. Lipid photosynthesis in olive fruit. Prog. Lipid Res. 33, 97-104.

Salvador MD, Aranda F, Fregapane G. 2001. Influence of fruit ripening on Cornicabra virgin olive oil quality. A study of four successive crop seasons. Food Chem. 73, 45-53.

Youssef NB, Zarrouk W, Carrasco-Pancorbo A Ouni Y, Segura-Carretero A, Fernández-Gutiérrez A, Daoud D, Zarrouk M. 2010. Effect of olive ripeness on chemicals propierties and phenolic composition of chetoui virgin olive oil. J. Sci. Food Agric. 90, 199-204.

Recibido: 9/05/12 Aceptado: 8/08/12 